\title{
IMPROVED HASHIN-SHTRIKMAN BOUNDS FOR ELASTIC MOMENT TENSORS AND AN APPLICATION
}

\author{
YVES CAPDEBOSCQ AND HYEONBAE KANG
}

\begin{abstract}
A Bstract. This paper is devoted to the derivation of trace bounds for elastic moment tensors. Starting from the integral equation formulation of the elastic moment tensor, we establish that its trace can be obtained as a sum of minimal energies. We then recover the so-called Hashin-Shtrikman bounds, and show that these bounds can be tightened for inclusions which have some local thickness. As an application, we show that the volume of the inclusion can be estimated by the elastic moment tensor.
\end{abstract}

\section{INTRODUCTION}

To each inclusion which occupies a bounded Lipschitz domain in $\mathbb{R}^{d}, d=2,3$, various geometric quantities are associated: eigenvalues and the capacity are among them. In this paper we deal with one of such quantities, namely, the elastic polarization tensor or the elastic moment tensor (EMT), which is a 4-tensor associated with elastic inclusions. This paper, in particular, is devoted to the derivation of optimal bounds for the trace of the EMT, and to the improvement of the bounds depending on the particular geometry of given inclusions.

As its name indicates the EMT is relevant in physics when studying the elastic properties of a given medium. There is an analogous notion in electromagnetism called the polarization tensor (PT). The PT is a 2-tensor or $d \times d$ matrix, and simpler to deal with. Thus, in order to motivate our work in this paper, we shall briefly review recent developments in the study of the PT and regard the present work as its analogy for the EMT.

Consider an inclusion $D$ in $\mathbb{R}^{d}$, a bounded Lipschitz domain, being inserted into a homogeneous medium of conductivity 1 in which there existed a uniform electric field $E=-a$, where $a$ is a constant vector. We assume that the conductivity of $D$ is $k \neq 1$. Due to the presence of the inclusion the uniform electric field is perturbed and the perturbed electric field is given by $E=-\nabla u$ where the potential $u$ is the solution to

$$
\begin{cases}\nabla \cdot\left(\left(1+(k-1) 1_{D}\right) \nabla u\right)=0 & \text { in } \mathbb{R}^{d}, \\ u(x)-a \cdot x=O\left(|x|^{1-d}\right) & \text { as }|x| \rightarrow \infty .\end{cases}
$$

Here and throughout this paper $1_{D}$ denotes the indicator function of $D$. The solution $u$ to (1.1) has a multipole asymptotic expansion at infinity, with a dipolar leading term:

$$
u(x)=a \cdot x+\frac{1}{\omega_{d}} \frac{\langle a, M x\rangle}{|x|^{d}}+O\left(|x|^{-d}\right), \quad \text { as }|x| \rightarrow \infty .
$$

2000 Mathematics Subject Classification. 35J50, 35R30, 35Q72, 74Q20.

corresponding author: Y. Capdeboscq, LMV, 45 avenue des États-Unis, 78035 Versailles Cedex, France. Tel: +331392546 40. Fax : +33139254645.

H.K. is partially supported by the grant KOSEF R01-2006-000-10002-0. 
Here $\omega_{d}$ is the area of the $d-1$ dimensional unit sphere and $M$ is a constant $d \times d$ matrix independent of $a$ and $x$. The matrix $M=M(D):=\left(M_{i j}\right)$ is called the polarization tensor associated with the inclusion $D$. We emphasize that $M$ depends only on the inclusion $D$ and conductivity contrast $k$. See [3, 24].

The concept of polarization tensor appears in various contexts such as the theory of homogenization $[20,24,26]$ (see [24] for an extensive list of references). In particular, the notion of PT appears as the low volume fraction limit of the effective properties of the dilute two phase composites $[8,21,22,25,27]$. This concept also appears in the context of inverse boundary value problems where it is used to detect diametrically small inclusions by means of boundary measurements. One can approximately detect, by boundary measurements, the location and the polarization tensor of the inclusion. Since the polarization tensor carries important geometric information, such as the volume of the inclusion, we are able to recover that information from boundary measurements. It was Friedman \& Vogelius [13] who first used the polarization tensor for the detection of small inclusions. After that there have been many significant development in this direction [2, 5, 9, 11]. We refer to recent books [3, 4] and references therein for recent developments of this theory.

Recently, the notion of PT associated with quite general type of inclusions has be defined [11, 12], together with upper and lower bounds for the trace of the PT based on the variational argument in [18]. The same bounds were also obtained by Lipton [20], for the low volume fraction limit of the homogenized conductivity. These bounds are known as Hashin-Shtrikman bounds in homogenization literature, see e.g. [24], after the names of the scientists who first found the optimal bounds on the effective conductivity of isotropic two-phase composites [14]. The PT bounds are given as follows: Let $|D|$ denote the volume of $D$. Then

$$
\operatorname{tr}(M) \leq|D|(k-1)\left(d-1+\frac{1}{k}\right),
$$

and

$$
|D| \operatorname{tr}\left(M^{-1}\right) \leq \frac{d-1+k}{k-1},
$$

where tr denotes the trace. It should be noted that the PT in this paper is $(k-1)$ times that in [11].

These bounds are optimal in two dimensions in the sense that any PT within the bounds is realized by a domain: by a coated ellipse [12] and a thin cross [1]. In both works, as the trace of the PT approaches to the upper bound (1.3), the corresponding domains get thinner. Thus a natural question is whether the upper bound can be improved if the domain has some 'thickness'. Since these estimates of the trace of the PT contains a significant information on estimation of the volume of the corresponding inclusion [12], this question has an important implication in estimation of the size of the inclusion; See the last section of this paper. This question for the improved Hashin-Shtrikman bounds for thick domains was answered affirmatively by the authors in [10]. It is worth noting that while the interior points of the Hashin-Shtrikman bounds can be realized by various shapes of inclusions, e.g., coated ellipses and various thin crosses [1], the lower bound (1.4) is rigid. It is proved in [16, 17] that if the lower bound is attained by a simply connected domain $D$, then $D$ must be an ellipse in two dimensions and an ellipsoid in three dimensions. As an immediate consequence, the Pólya-Szegö conjecture [28], which asserts that the inclusion whose PT has the minimal trace takes the shape of a disk or a ball, has been proved. 
While the PT describes the dipole expansion of the perturbation of the electric field due to the presence of the inclusion, the EMT does that for the elastic field. The precise definition of the EMT will be given in the next section. The EMT was introduced by Maz'ya and Nazarov in relation to the asymptotic expansion for energy due to existence of a small hole or cavity [23]. See also [19, 26] for various applications of EMT. The definition of EMT has been extended to include the case when inclusions are not holes or hard ones and used for reconstruction of unknown inclusions via boundary measurements $[7,15]$.

In this paper, we first derive bounds of Hashin-Shtrikman type for the EMT. The exact Hashin-Shtrikman bounds are not new: they were already derived by Lipton [20]. We show here that an additional term can be added to the upper bounds, which is especially relevant when some general information is known about the shape of the inclusions. We show for example that when the inclusion has some 'thickness', this additional term leads us to an explicit strict upper bound. We define the thickness of a domain $D$ as a dimensionless number $\tau \in] 0,1]$ given by

$$
\tau=\max \frac{|D|}{\left|B_{r}(x)\right|}
$$

where the maximum is taken over all $r>0$ and $x \in \mathbb{R}^{d}$ such that $D \subset B_{r}(x)$. Here and throughout this paper $B_{r}(x)$ denotes the open ball of radius $r$ centered at $x$. Note that $\tau=1$ for and only for the balls, and that $\tau$ is close to zero for very thin domains. For example, for an ellipsoid with $d-1$ major axis equal to $a$ and a minor axis equal to $b<a$, $\tau=b / a$.

This paper is organized as follows. In the next section we set up some notation and review basic facts on the Layer potentials. We then review some results on the EMT from [7] and show that the EMT can be obtained a sum of minimal energies. Section 3 is devoted to derivation of pointwise bounds for EMT, while section 4 is for Hashin-Shtrikman bounds with additional terms. In section 5 , we estimate this additional term in terms of the thickness of the inclusion. We then conclude the paper with an application of the bounds for EMT to the estimation of the volume of the inclusion.

\section{Elastic Moment Tensor}

We begin by introducing some common notation. In $\mathbb{R}^{d}, d=2,3$, let

$$
\begin{aligned}
& \mathbf{I}_{2}:=\delta_{i j} \mathbf{e}_{i} \otimes \mathbf{e}_{j}, \\
& \mathbf{I}_{4}:=\frac{1}{2}\left(\delta_{i k} \delta_{j l}+\delta_{i l} \delta_{j k}\right) \mathbf{e}_{i} \otimes \mathbf{e}_{j} \otimes \mathbf{e}_{k} \otimes \mathbf{e}_{l} .
\end{aligned}
$$

Here and throughout this paper the Einstein convention for summation is used. $\mathbf{I}_{2}$ is $d \times d$ identity matrix or 2-tensor while $\mathbf{I}_{4}$ is the identity 4 -tensor. The following notations are also convenient:

$$
\boldsymbol{\Lambda}_{1}:=\frac{1}{d} \mathbf{I}_{2} \otimes \mathbf{I}_{2}, \quad \boldsymbol{\Lambda}_{2}:=\mathbf{I}_{4}-\boldsymbol{\Lambda}_{1} .
$$

Let $a: b$ denote the contraction of two matrices $a$ and $b$, i.e., $a: b=a_{i j} b_{i j}=\operatorname{tr}\left(a^{T} b\right)$ where $\operatorname{tr}(a)$ denotes the trace of $a$ and $a^{T}$ denotes the transpose of $a$. Since for any $d \times d$ symmetric matrix $a$

$$
\mathbf{I}_{2} \otimes \mathbf{I}_{2}(a)=\left(a: \mathbf{I}_{2}\right) \mathbf{I}_{2}=\operatorname{trace}(a) \mathbf{I}_{2}
$$

and

$$
\mathbf{I}_{4}(a)=a,
$$


one can immediately see that

$$
\boldsymbol{\Lambda}_{1} \boldsymbol{\Lambda}_{1}=\boldsymbol{\Lambda}_{1}, \quad \boldsymbol{\Lambda}_{2} \boldsymbol{\Lambda}_{2}=\boldsymbol{\Lambda}_{2}, \quad \boldsymbol{\Lambda}_{1} \boldsymbol{\Lambda}_{2}=0 .
$$

Let $D$ be a bounded Lipschitz domain in $\mathbb{R}^{d}$ which occupies an isotropic elastic body with the Lamé constants $(\lambda, \mu)$ satisfying

$$
\mu>0 \text { and } d \lambda+2 \mu>0 .
$$

Then the elasticity tensor for $D$ is given by

$$
C:=\lambda \mathbf{I}_{2} \otimes \mathbf{I}_{2}+2 \mu \mathbf{I}_{4}=d \kappa \boldsymbol{\Lambda}_{\mathbf{1}}+2 \mu \boldsymbol{\Lambda}_{\mathbf{2}},
$$

where $\kappa=\lambda+2 \mu / d$ denotes the bulk modulus of $D$. For a given displacement vector $\mathbf{u}$, the strain is defined to be

$$
\mathcal{E}(\mathbf{u}):=\frac{1}{2}\left(\nabla \mathbf{u}+\nabla \mathbf{u}^{T}\right) .
$$

The elastostatic system corresponding to the Lamé constants $\lambda, \mu$ is defined by

$$
\mathcal{L}_{C} \mathbf{u}:=\nabla \cdot(C \mathcal{E}(\mathbf{u}))=\mu \Delta \mathbf{u}+(\lambda+\mu) \nabla \nabla \cdot \mathbf{u},
$$

and the corresponding co-normal derivative $\partial \mathbf{u} / \partial \nu$ on $\partial D$ is defined to be

$$
\frac{\partial \mathbf{u}}{\partial \nu}:=C \mathcal{E}(\mathbf{u}) N=\lambda(\nabla \cdot \mathbf{u}) N+\mu\left(\nabla \mathbf{u}+\nabla \mathbf{u}^{T}\right) N \quad \text { on } \partial D,
$$

where $N$ is the outward unit normal to $\partial D$.

The Kelvin matrix of fundamental solutions $\boldsymbol{\Gamma}=\left(\Gamma_{i j}\right)_{i, j=1}^{d}$ for the Lamé system $\mathcal{L}_{C}$ is defined to be

$$
\Gamma_{i j}(x):=A \Gamma_{0}(x) \delta_{i j}-B x_{j} \frac{\partial \Gamma_{0}}{\partial x_{i}}(x), \quad x \neq 0,
$$

where $\Gamma_{0}$ is the fundamental solution for the Laplacian, i.e.,

$$
\Gamma_{0}(x)= \begin{cases}\frac{1}{2 \pi} \log |x|, & \text { if } d=2, \\ -\frac{1}{4 \pi} \frac{1}{|x|}, & \text { if } d=3,\end{cases}
$$

and

$$
A=\frac{1}{2}\left(\frac{1}{\mu}+\frac{1}{2 \mu+\lambda}\right), \quad B=\frac{1}{2}\left(\frac{1}{\mu}-\frac{1}{2 \mu+\lambda}\right) .
$$

The single layer potentials of the density function $\varphi$ on $D$ associated with the Lamé parameters $(\lambda, \mu)$ are defined by

$$
\mathcal{S}[\varphi](x):=\int_{\partial D} \boldsymbol{\Gamma}(x-y) \varphi(y) d \sigma(y), \quad x \in \mathbb{R}^{d} .
$$

Let $D$ be a bounded Lipschitz domain in $\mathbb{R}^{d}$. Let $C^{0}:=\lambda \mathbf{I}_{2} \otimes \mathbf{I}_{2}+2 \mu \mathbf{I}_{4}$ be the elasticity tensor of $\mathbb{R}^{d} \backslash \bar{D}$ and $C^{1}:=\tilde{\lambda} \mathbf{I}_{2} \otimes \mathbf{I}_{2}+2 \tilde{\mu} \mathbf{I}_{4}$ be that of $D$. Let $\kappa$ and $\tilde{\kappa}$ be bulk modulus of $\mathbb{R}^{d} \backslash \bar{D}$ and $D$, respectively. It is always assumed that

$$
\mu>0, \quad d \lambda+2 \mu>0, \quad \tilde{\mu}>0 \quad \text { and } \quad d \tilde{\lambda}+2 \tilde{\mu}>0 .
$$

We also assume that

$$
(\lambda-\tilde{\lambda})(\mu-\tilde{\mu})>0
$$


in other words, either $C^{1}>C^{0}$ or $C^{1}<C^{0}$. Let $\mathcal{L}_{C^{1}}$ and $\frac{\partial u}{\partial \tilde{\nu}}$ be the Lamé system and the co-normal derivative associated with $(\tilde{\lambda}, \tilde{\mu})$, respectively. We also denote the single layer potential corresponding to $(\tilde{\lambda}, \tilde{\mu})$ on $D$ by $\widetilde{\mathcal{S}}$.

In [7], the first order elastic moment tensor is defined as follows:

Definition 2.1 (Elastic moment tensors). For $i, j=1, \ldots, d$, let $\left(\mathbf{f}_{i j}, \mathbf{g}_{i j}\right)$ be the solution of the integral equation

$$
\left\{\begin{array}{l}
\left.\widetilde{\mathcal{S}}\left[\mathbf{f}_{i j}\right]\right|_{-}-\left.\mathcal{S}\left[\mathbf{g}_{i j}\right]\right|_{+}=x_{i} \mathbf{e}_{j} \\
\left.\frac{\partial}{\partial \widetilde{\mathcal{S}}} \widetilde{\mathcal{S}}\left[\mathbf{f}_{i j}\right]\right|_{-}-\left.\frac{\partial}{\partial \nu} \mathcal{S}\left[\mathbf{g}_{i j}\right]\right|_{+}=\frac{\partial\left(x_{i} \mathbf{e}_{j}\right)}{\partial \nu} \quad \text { on } \partial D,
\end{array}\right.
$$

where the subscripts + and - denote the limit from outside and inside $D$, respectively. Then, the EMT $M=\left(m_{i j p q}\right)$ associated with $D$ and $\lambda, \mu, \tilde{\lambda}, \tilde{\mu}$ is defined by

$$
m_{i j p q}=\int_{\partial D} x_{p} \mathbf{e}_{q} \cdot \mathbf{g}_{i j} d \sigma, \quad i, j, p, q=1, \ldots, d .
$$

We note that this tensor is exactly the one defined by Maz'ya and Nazarov for holes (see [7]). Moreover, the definition (2.9) covers the case when $\tilde{\mu}$ is nonzero and finite.

Let

$$
C_{D}:=\left(1-1_{D}\right) C^{0}+1_{D} C^{1},
$$

where $1_{D}$ is the indicator function for $D$. For a given $d \times d$ symmetric matrix $a$, let $u$ be the solution to

$$
\left\{\begin{array}{l}
\nabla \cdot\left(C_{D} \mathcal{E}(\mathbf{u})\right)=0 \quad \text { in } \mathbb{R}^{d}, \\
\mathbf{u}(x)-a x=O\left(|x|^{1-d}\right) \text { as }|x| \rightarrow \infty .
\end{array}\right.
$$

Then $\nabla \mathbf{u}$ represents the elastic field perturbed due to the presence of the inclusion $D$ under the uniform loading given by $\nabla(a x)$. It is known [7] that the solution $\mathbf{u}$ has the following asymptotic expansion:

$$
\mathbf{u}(x)=a x+\sum_{i, j, p, q=1}^{d} a_{i j} \partial_{p} \Gamma_{k q}(x) m_{i j p q}+O\left(|x|^{-d}\right) \quad \text { as }|x| \rightarrow \infty .
$$

The EMT also appears in the low volume fraction limit of the effective elastic property [6].

The following identity is proved in [7].

Proposition 2.2. Suppose that $\mu \neq \tilde{\mu}$. Given a nonzero symmetric matrix $a=\left(a_{i j}\right)$, define $\varphi_{a}, \mathbf{f}_{a}$, and $\mathbf{g}_{a} b y$

$$
\varphi_{a}:=\left(a_{i j}\right) x=a_{i j} x_{j} \mathbf{e}_{i}, \quad \mathbf{f}_{a}:=a_{i j} \mathbf{f}_{i j}, \quad \mathbf{g}_{a}:=a_{i j} \mathbf{g}_{i j} .
$$

Let

$$
P(a):=\frac{\tilde{\kappa}+\kappa}{\tilde{\kappa}-\kappa} \boldsymbol{\Lambda}_{1}(a)+\frac{\tilde{\mu}+\mu}{\tilde{\mu}-\mu} \boldsymbol{\Lambda}_{2}(a) .
$$

Then,

$$
P a: M a=\int_{D} C^{1} \mathcal{E}\left(\widetilde{\mathcal{S}} \mathbf{f}_{a}\right): \mathcal{E}\left(\widetilde{\mathcal{S}} \mathbf{f}_{a}\right)+\int_{\mathbb{R}^{d} \backslash \bar{D}} C^{0} \mathcal{E}\left(\mathcal{S} \mathbf{g}_{a}\right): \mathcal{E}\left(\mathcal{S} \mathbf{g}_{a}\right)+\int_{D} C^{0} \mathcal{E}\left(\varphi_{a}\right): \mathcal{E}\left(\varphi_{a}\right)
$$


As a consequence of Proposition 2.2, the following symmetry is obtained in [7]:

$$
m_{i j p q}=m_{i j q p}, \quad m_{i j p q}=m_{j i p q}, \quad \text { and } \quad m_{i j p q}=m_{p q i j}, \quad i, j, p, q=1, \ldots, d .
$$

The symmetry (2.16) implies that $M$ is a symmetric linear transformation on the space $M_{d}^{S}$ of $d \times d$ symmetric matrices. It is also proved that $M$ is positive-definite if $\tilde{\lambda}>\lambda$ and $\tilde{\mu}>\mu$, and negative-definite if $\tilde{\lambda}<\lambda$ and $\tilde{\mu}<\mu$. Let us assume that $\tilde{\lambda}>\lambda$ and $\tilde{\mu}>\mu$ for convenience. Let $k_{j}, j=1, \ldots, N_{d}:=\frac{d(d+1)}{2}$, be eigenvalues of $M$ on $M_{d}^{S}$ and $A_{j}$ be the corresponding eigen-matrix of the unit length. Then, $M$ can be written as

$$
M=\sum_{j=1}^{N_{d}} k_{j} A_{j} \otimes A_{j} .
$$

Lemma 2.3. For any $a \in M_{d}^{S}$,

(i) $\boldsymbol{\Lambda}_{1} a: M a=a: \boldsymbol{\Lambda}_{1} M a=a: M \boldsymbol{\Lambda}_{1} a$.

(ii) $\boldsymbol{\Lambda}_{2} a: M a=a: \boldsymbol{\Lambda}_{2} M a=a: M \boldsymbol{\Lambda}_{2} a$.

Proof. Because of (2.17), we may assume that $M=A \otimes A$ for some $A \in M_{d}^{S}$. We first get

$$
\boldsymbol{\Lambda}_{1} a: M a=\frac{\operatorname{tr}(a)}{d}\left(\mathbf{I}_{2}: M a\right)=\frac{1}{d} \operatorname{tr}(a) \operatorname{tr}(M a)=a: \boldsymbol{\Lambda}_{1} M a .
$$

On the other hand, since $M a=(A: a) A$, we get

$$
\mathbf{\Lambda}_{1} M a=(A: a)\left(A: \mathbf{I}_{2}\right) \mathbf{I}_{2} .
$$

It then follows that

$$
a: \boldsymbol{\Lambda}_{1} M a=(A: a)\left(A: \mathbf{I}_{2}\right)\left(\mathbf{I}_{2}: a\right)=a:\left(\left(A: \mathbf{I}_{2}\right)\left(\mathbf{I}_{2}: a\right) A\right)=a: M \boldsymbol{\Lambda}_{1} a .
$$

This proves (i). (ii) follows immediately from (i) and the proof is complete.

\section{A PAIR OF ENERGY AND GEOMETRY INDEPENDENT POINTWISE BOUNDS}

Observe that $\varphi_{a}, \mathbf{f}_{a}, \mathbf{g}_{a}$ in (2.13) satisfy the following relation:

$$
\left\{\begin{array}{l}
\left.\widetilde{\mathcal{S}} \mathbf{f}_{a}\right|_{-}-\left.\mathcal{S} \mathbf{g}_{a}\right|_{+}=\varphi_{a} \\
\left.\left.\frac{\partial}{\partial \widetilde{\nu}} \widetilde{\mathcal{S}}\right|_{a}\right|_{-}-\left.\frac{\partial}{\partial \nu} \mathcal{S} \mathbf{g}_{a}\right|_{+}=\frac{\partial \varphi_{a}}{\partial \nu} \quad \text { on } \partial D .
\end{array}\right.
$$

Define $\mathbf{v}$ by

$$
\mathbf{v}(x):= \begin{cases}\widetilde{\mathcal{S}} \mathbf{f}_{a}(x)-\varphi_{a}(x), & x \in D, \\ \mathcal{S} \mathbf{g}_{a}(x), & x \in \mathbb{R}^{d} \backslash \bar{D} .\end{cases}
$$

Then one can see from (3.1) that $\mathbf{v}$ satisfies the following transmission condition along $\partial D$ :

$$
\left.\mathbf{v}\right|_{-}-\left.\mathbf{v}\right|_{+}=0 \text { and }\left.\quad \frac{\partial \mathbf{v}}{\partial \widetilde{\nu}}\right|_{-}-\left.\frac{\partial \mathbf{v}}{\partial \nu}\right|_{+}=\left.\frac{\partial \varphi_{a}}{\partial \widetilde{\nu}}\right|_{-}-\left.\frac{\partial \varphi_{a}}{\partial \nu}\right|_{+} \quad \text { on } \partial D,
$$

and it is the unique solution to the following transmission problem for the elastostatic system:

$$
\left\{\begin{array}{l}
\nabla \cdot\left(C_{D} \mathcal{E}\left(\mathbf{v}+\varphi_{a}\right)\right)=0 \text { in } \mathbb{R}^{d} \\
\mathbf{v}(x)=O\left(|x|^{-1}\right) \text { as }|x| \rightarrow \infty
\end{array}\right.
$$


Note that $\mathbf{v}(x)=O\left(|x|^{1-d}\right)$ as $|x| \rightarrow \infty$ because $\int_{\partial D} \mathbf{g}_{a} d \sigma=0$. Writing (3.3) in variational form, we obtain

$$
\int_{\mathbb{R}^{d}} C_{D}\left(\mathcal{E}(\mathbf{v})+1_{D}\left(\mathbf{I}_{4}-\left(C^{1}\right)^{-1} C^{0}\right) a\right): \mathcal{E}(\mathbf{w}) d x=0
$$

for any $\mathbf{w} \in H^{1}\left(\mathbb{R}^{d}\right)$, and hence $\mathbf{v}$ is the minimizer in $H^{1}\left(\mathbb{R}^{d}\right)$ of the energy form

$$
\int_{\mathbb{R}^{d}} C_{D}\left(\mathcal{E}(\mathbf{v})+1_{D}\left(\mathbf{I}_{4}-\left(C^{1}\right)^{-1} C^{0}\right) a\right):\left(\mathcal{E}(\mathbf{v})+1_{D}\left(\mathbf{I}_{4}-\left(C^{1}\right)^{-1} C^{0}\right) a\right) d x .
$$

Let

$$
G:=\mathbf{I}_{4}-\left(C^{1}\right)^{-1} C^{0} .
$$

Observe that $G$ is invertible on $M_{d}^{S}$. In fact, we have

$$
G^{-1}=\left(C^{1}-C^{0}\right)^{-1} C^{1} \text {. }
$$

We can now rewrite $(2.15)$ as

$$
a: P M a=\int_{\mathbb{R}^{d}} C_{D}\left(\mathcal{E}(\mathbf{v})+1_{D} a\right):\left(\mathcal{E}(\mathbf{v})+1_{D} a\right) d x+\left(C^{0} a: a\right)|D| .
$$

We now express the identity (3.8) as a sum of minimal energies. To this end, we introduce the following notation: for a domain $D$ and $d \times d$ symmetric matrix $a$, let

$$
E(D, a)=\min _{\mathbf{v} \in H^{1}\left(\mathbb{R}^{d}\right)^{d}} \int_{\mathbb{R}^{d}} C_{D}\left(\mathcal{E}(\mathbf{v})+1_{D} G a\right):\left(\mathcal{E}(\mathbf{v})+1_{D} G a\right) d x,
$$

where $\mathbf{v}$ is defined by (3.1) and (3.2).

Proposition 3.1. The EMT M satisfies the following identity:

$$
\begin{aligned}
a: P M a= & \frac{1}{2}\left(\frac{\tilde{\kappa}+\kappa}{\tilde{\kappa}-\kappa}+\frac{\tilde{\mu}+\mu}{\tilde{\mu}-\mu}\right) E(D, a) \\
& +\frac{1}{2}\left(\frac{\tilde{\kappa}+\kappa}{\tilde{\kappa}-\kappa}-\frac{\tilde{\mu}+\mu}{\tilde{\mu}-\mu}\right)\left(E\left(D, \boldsymbol{\Lambda}_{1}(a)\right)-E\left(D, \boldsymbol{\Lambda}_{2}(a)\right)\right) \\
& +|D|\left(C^{0}+C^{1}\right)\left(C^{1}\right)^{-1} C^{0} a: a .
\end{aligned}
$$

Proof. Let $a_{j}=\boldsymbol{\Lambda}_{j}(a), j=1,2$, and define $\mathbf{v}_{j}$ by (3.1) and (3.2) with $a$ replaced with $a_{j}$. Then by linearity, we have $\mathbf{v}=\mathbf{v}_{1}+\mathbf{v}_{2}$. Decompose $G$ uniquely as

$$
G=g_{1} \boldsymbol{\Lambda}_{1}+g_{2} \boldsymbol{\Lambda}_{2} \text {. }
$$

We then have

$$
\begin{aligned}
& \int_{\mathbb{R}^{d}} C_{D}\left(\mathcal{E}(\mathbf{v})+1_{D} a\right):\left(\mathcal{E}(\mathbf{v})+1_{D} a\right) d x \\
& =\sum_{i, j=1}^{2}\left(\int_{\mathbb{R}^{d}} C_{D} \mathcal{E}\left(\mathbf{v}_{i}\right): \mathcal{E}\left(\mathbf{v}_{j}\right) d x+2 \int_{\mathbb{R}^{d}} C_{D} 1_{D} a_{i}: \mathcal{E}\left(\mathbf{v}_{j}\right) d x\right)+\left(C^{1} a: a\right)|D| \\
& =\sum_{i, j=1}^{2}\left(\int_{\mathbb{R}^{d}} C_{D} \mathcal{E}\left(\mathbf{v}_{i}\right): \mathcal{E}\left(\mathbf{v}_{j}\right) d x+\frac{2}{g_{i}} \int_{\mathbb{R}^{d}} C_{D} 1_{D} G a_{i}: \mathcal{E}\left(\mathbf{v}_{j}\right) d x\right)+\left(C^{1} a: a\right)|D| .
\end{aligned}
$$

Since $\mathbf{v}_{i}$ is the minimizer of the energy

$$
\int_{\mathbb{R}^{d}} C_{D}\left(\mathcal{E}\left(\mathbf{v}_{i}\right)+1_{D} G a_{i}\right):\left(\mathcal{E}\left(\mathbf{v}_{i}\right)+1_{D} G a_{i}\right) d x
$$


for $i=1,2$, we have

$$
\int_{\mathbb{R}^{d}} C_{D} 1_{D} G a_{i}: \mathcal{E}\left(\mathbf{v}_{j}\right) d x=-\int_{\mathbb{R}^{d}} C_{D} \mathcal{E}\left(\mathbf{v}_{i}\right): \mathcal{E}\left(\mathbf{v}_{j}\right) d x, \quad i, j=1,2 .
$$

This in turn yields

$$
\begin{aligned}
& \int_{\mathbb{R}^{d}} C_{D}\left(\mathcal{E}(\mathbf{v})+1_{D} a\right):\left(\mathcal{E}(\mathbf{v})+1_{D} a\right) d x \\
& =\sum_{i, j=1}^{2}\left(1-\frac{2}{g_{i}}\right) \int_{\mathbb{R}^{d}} C_{D} \mathcal{E}\left(\mathbf{v}_{i}\right): \mathcal{E}\left(\mathbf{v}_{j}\right) d x+\left(C^{1} a: a\right)|D| \\
& =-\left(\frac{1}{g_{1}}-\frac{1}{g_{2}}\right) \int_{\mathbb{R}^{d}} C_{D} \mathcal{E}\left(\mathbf{v}_{1}\right): \mathcal{E}\left(\mathbf{v}_{1}\right) d x-\left(\frac{1}{g_{2}}-\frac{1}{g_{1}}\right) \int_{\mathbb{R}^{d}} C_{D} \mathcal{E}\left(\mathbf{v}_{2}\right): \mathcal{E}\left(\mathbf{v}_{2}\right) d x \\
& \quad-\left(\frac{1}{g_{1}}+\frac{1}{g_{2}}-1\right) \int_{\mathbb{R}^{d}} C_{D} \mathcal{E}(\mathbf{v}): \mathcal{E}(\mathbf{v}) d x+\left(C^{1} a: a\right)|D|
\end{aligned}
$$

Using again the minimizing properties of $\mathbf{v}_{1}, \mathbf{v}_{2}$ and $\mathbf{v}=\mathbf{v}_{1}+\mathbf{v}_{2}$, we get

$$
\begin{aligned}
& \int_{\mathbb{R}^{d}} C_{D}\left(\mathcal{E}(\mathbf{v})+1_{D} G a\right):\left(\mathcal{E}(\mathbf{v})+1_{D} G a\right) d x \\
& =-\int_{\mathbb{R}^{d}} C_{D} \mathcal{E}(\mathbf{v}): \mathcal{E}(\mathbf{v}) d x+\int_{\mathbb{R}^{d}} C_{D}\left(1_{D} G a\right):\left(1_{D} G a\right) d x
\end{aligned}
$$

and the same identities with $\mathbf{v}$ and $a$ replaced with $\mathbf{v}_{j}$ and $a_{j}, j=1,2$. Since $C^{1}$ is isotropic, $\boldsymbol{\Lambda}_{i} C^{1} \boldsymbol{\Lambda}_{j}=0$ if $i \neq j$, and hence

$$
C^{1} a: a=C^{1} a_{1}: a_{1}+C^{1} a_{2}: a_{2} .
$$

It then follows that

$$
\begin{aligned}
& \int_{\mathbb{R}^{d}} C_{D}\left(\mathcal{E}(\mathbf{v})+1_{D} a\right):\left(\mathcal{E}(\mathbf{v})+1_{D} a\right) d x \\
& =\left(\frac{1}{g_{1}}+\frac{1}{g_{2}}-1\right) E(D, a)+\left(\frac{1}{g_{1}}-\frac{1}{g_{2}}\right)\left(E\left(D, \boldsymbol{\Lambda}_{1}(a)\right)-E\left(D, \mathbf{\Lambda}_{2}(a)\right)\right) \\
& \quad+\left(g_{1}-1\right)^{2} C^{1} a_{1}: a_{1}|D|+\left(g_{2}-1\right)^{2} C^{1} a_{2}: a_{2}|D| .
\end{aligned}
$$

Finally, note that

$$
\begin{aligned}
\sum_{i=1}^{2}\left(g_{i}-1\right)^{2} C^{1} a_{i}: a_{i} & =\sum_{i=1}^{2}\left(g_{i}-1\right)^{2} \boldsymbol{\Lambda}_{i} C^{1} \boldsymbol{\Lambda}_{i} a: a \\
& =\left(G C^{1} G-2 C^{1} G+C^{1}\right) a: a \\
& =\left(C^{0}\right)^{2}\left(C^{1}\right)^{-1} a: a .
\end{aligned}
$$

Since

and

$$
g_{1}^{-1}-g_{2}^{-1}=\frac{1}{2} \frac{\tilde{\kappa}+\kappa}{\tilde{\kappa}-\kappa}-\frac{1}{2} \frac{\tilde{\mu}+\mu}{\tilde{\mu}-\mu}
$$

$$
g_{1}^{-1}+g_{2}^{-1}-1=\frac{1}{2} \frac{\tilde{\kappa}+\kappa}{\tilde{\kappa}-\kappa}+\frac{1}{2} \frac{\tilde{\mu}+\mu}{\tilde{\mu}-\mu}
$$

and thanks to (3.8) we obtain (3.10), which completes the proof. 
Note that

$$
\begin{aligned}
& \left(C^{0}+C^{1}\right)\left(C^{1}\right)^{-1} C^{0}=d(\tilde{\kappa}+\kappa) \frac{\kappa}{\tilde{\kappa}} \boldsymbol{\Lambda}_{1}+2(\tilde{\mu}+\mu) \frac{\mu}{\tilde{\mu}} \boldsymbol{\Lambda}_{2}, \\
& \left(C^{1}-C^{0}\right)\left(C^{1}\right)^{-1} C^{0}=d(\tilde{\kappa}-\kappa) \frac{\kappa}{\tilde{\kappa}} \boldsymbol{\Lambda}_{1}+2(\tilde{\mu}-\mu) \frac{\mu}{\tilde{\mu}} \boldsymbol{\Lambda}_{2} .
\end{aligned}
$$

Therefore, we can write in condensed form

$$
\begin{aligned}
& \frac{\tilde{\kappa}-\kappa}{\tilde{\kappa}+\kappa}\left(C^{0}+C^{1}\right)\left(C^{1}\right)^{-1} C^{0} \boldsymbol{\Lambda}_{1}=\left(C^{1}-C^{0}\right)\left(C^{1}\right)^{-1} C^{0} \boldsymbol{\Lambda}_{1}, \\
& \frac{\tilde{\mu}-\mu}{\tilde{\mu}+\mu}\left(C^{0}+C^{1}\right)\left(C^{1}\right)^{-1} C^{0} \boldsymbol{\Lambda}_{2}=\left(C^{1}-C^{0}\right)\left(C^{1}\right)^{-1} C^{0} \boldsymbol{\Lambda}_{2},
\end{aligned}
$$

and we obtain the following corollary from (2.14).

Corollary 3.2. If $a=\Lambda_{1}(a)$ or $a=\Lambda_{2}(a)$, we have

$$
\begin{aligned}
a: M a= & \min _{\mathbf{v} \in H^{1}\left(\mathbb{R}^{d}\right)} \int_{\mathbb{R}^{d}} C_{D}\left(\mathcal{E}(\mathbf{v})+1_{D} G a\right):\left(\mathcal{E}(\mathbf{v})+1_{D} G a\right) d x \\
& +|D|\left(C^{1}-C^{0}\right)\left(C^{1}\right)^{-1} C^{0} a: a .
\end{aligned}
$$

The energy formulation (3.14), yields a priori bounds on $M$.

Corollary 3.3. The following bounds hold

$$
d|D|(\tilde{\kappa}-\kappa) \frac{\kappa}{\tilde{\kappa}} \boldsymbol{\Lambda}_{1} \leq \boldsymbol{\Lambda}_{1} M \boldsymbol{\Lambda}_{1} \leq d|D|(\tilde{\kappa}-\kappa) \boldsymbol{\Lambda}_{1},
$$

and

$$
2|D|(\tilde{\mu}-\mu) \frac{\mu}{\tilde{\mu}} \boldsymbol{\Lambda}_{2} \leq \boldsymbol{\Lambda}_{2} M \boldsymbol{\Lambda}_{2} \leq 2|D|(\tilde{\mu}-\mu) \boldsymbol{\Lambda}_{2} .
$$

Note that upper bounds in (3.15) and (3.16) are obtained by the choice $\mathbf{v}=0$ in (3.14). We emphasize that (3.15) and (3.16) hold even if $C^{1}<C^{0}$.

\section{Hashin-Shtrikman Bounds with an additional term}

In this section we shall use the so-called Hashin-Shtrikman variational method, as described by Kohn \& Milton in [18], to prove inequalities on the 4-tensor $\widetilde{M}$ defined by

$$
\begin{aligned}
a: \widetilde{M} a= & \min _{\mathbf{v} \in H^{1}\left(\mathbb{R}^{d}\right)} \int_{\mathbb{R}^{d}} C_{D}\left(\mathcal{E}(\mathbf{v})+1_{D} G a\right):\left(\mathcal{E}(\mathbf{v})+1_{D} G a\right) d x \\
& +|D|\left(C^{1}-C^{0}\right)\left(C^{1}\right)^{-1} C^{0} a: a .
\end{aligned}
$$

With the help of Proposition 3.1 and Corollary 3.2 this will in turn yield bounds on the EMT, since $M=\widetilde{M}$ if $a=\boldsymbol{\Lambda}_{1}(a)$ or $a=\boldsymbol{\Lambda}_{2}(a)$.

Let us first define an operator which will be used afterwards. For $w \in L^{2}\left(\mathbb{R}^{d}: M_{d}^{S}\right)$, let $\Phi$ be the unique solution in $H^{1}\left(\mathbb{R}^{d}: M_{d}^{S}\right)$ to

$$
\mathcal{L}_{C}(\Phi)=\nabla \cdot w
$$

and then define the linear operator $F_{C}$ by

$$
F_{C}(w):=\mathcal{E}(\Phi) .
$$

In other words, $F_{C}$ is defined by

$$
F_{C}(w):=\mathcal{E L}_{C}^{-1}(\nabla \cdot w)
$$


As a first step in deriving the trace bounds of EMT, we see, by comparing the background medium $C_{0}$ with a reference medium, that the energy formula (3.9) can be reformulated as a minimum or a maximum of a certain functional, as it is explained by the following proposition.

Proposition 4.1. Let $\widetilde{M}$ be given by formula (4.1). Let $C$ denote a constant (isotropic) elasticity tensor given by $C=2 \mu_{c} \mathbf{I}_{4}+\lambda_{c} \mathbf{I}_{2} \otimes \mathbf{I}_{2}$. For any such tensor $C<C_{D}$ we have,

$$
a: \widetilde{M} a=|D| a:\left(C^{1}-C^{0}\right)\left(C-C^{1}\right)^{-1}\left(C-C^{0}\right) a+\sup _{\sigma \in L^{2}\left(\mathbb{R}^{d}: M_{d}^{S}\right)} W_{a}(C, \sigma),
$$

where

$$
W_{a}(C, \sigma)=\int_{\mathbb{R}^{d}} \sigma: F_{C} \sigma+\int_{\mathbb{R}^{d}}\left(C-C_{D}\right)^{-1} \sigma: \sigma+2 \int_{D} \sigma:\left(C^{1}-C\right)^{-1}\left(C^{1}-C^{0}\right) a .
$$

If $C>C_{D}$, we have

$$
a: \widetilde{M} a=|D| a:\left(C^{1}-C^{0}\right)\left(C-C^{1}\right)^{-1}\left(C-C^{0}\right) a+\inf _{\sigma \in L^{2}\left(\mathbb{R}^{d}: M_{d}^{S}\right)} W_{a}(C, \sigma) .
$$

Proof. We give a proof only when $C^{1}>C^{0}$. Let $C$ be a constant elasticity tensor given by $C=2 \mu_{c} \mathbf{I}_{4}+\lambda_{c} \mathbf{I}_{2} \otimes \mathbf{I}_{2}$ as before and suppose that $C<C_{D}$. Then we have

$$
\begin{aligned}
& \int_{\mathbb{R}^{d}} C_{D}\left(\mathcal{E}(\mathbf{v})+1_{D} G a\right):\left(\mathcal{E}(\mathbf{v})+1_{D} G a\right) d x \\
& =\int_{\mathbb{R}^{d}}\left(C_{D}-C\right)\left(\mathcal{E}(\mathbf{v})+1_{D} G a\right):\left(\mathcal{E}(\mathbf{v})+1_{D} G a\right) d x \\
& \quad+\int_{\mathbb{R}^{d}} C\left(\mathcal{E}(\mathbf{v})+1_{D} G a\right):\left(\mathcal{E}(\mathbf{v})+1_{D} G a\right) d x
\end{aligned}
$$

Using the positivity of $C_{D}-C$ and the convex duality, the first term in the right-hand-side of the above identity can be written as

$$
\sup _{\eta \in L^{2}\left(\mathbb{R}^{d}: M_{d}^{S}\right)}\left[-\int_{\mathbb{R}^{d}}\left(C_{D}-C\right)^{-1} \eta: \eta d x+2 \int_{\mathbb{R}^{d}} \eta:\left(\mathcal{E}(\mathbf{v})+1_{D} G a\right) d x\right] .
$$

Interchanging the order of the minimization and maximization (see [18]), we obtain

$$
\begin{aligned}
& \min _{\mathbf{v} \in H^{1}\left(\mathbb{R}^{d}\right)} \int_{\mathbb{R}^{d}} C_{D}\left(\mathcal{E}(\mathbf{v})+1_{D} G a\right):\left(\mathcal{E}(\mathbf{v})+1_{D} G a\right) d x \\
= & \sup _{\eta \in L^{2}\left(\mathbb{R}^{d}: M_{d}^{S}\right)}\left[-\int_{\mathbb{R}^{d}}\left(C_{D}-C\right)^{-1} \eta: \eta-\int_{\mathbb{R}^{d}} C^{-1} \eta: \eta\right. \\
& \left.+\inf _{\mathbf{v} \in H^{1}\left(\mathbb{R}^{d}\right)} \int_{\mathbb{R}^{d}} C\left(\mathcal{E}(\mathbf{v})+1_{D} G a+C^{-1} \eta\right):\left(\mathcal{E}(\mathbf{v})+1_{D} G a+C^{-1} \eta\right)\right] .
\end{aligned}
$$

One can easily see that the infimum of the last term in (4.6) is attained by the solution in $H^{1}\left(\mathbb{R}^{d}: M_{d}^{S}\right)$ of

i.e., by

$$
-\nabla \cdot(C \mathcal{E}(\mathbf{v}))=\nabla \cdot\left(1_{D} C G_{a}+\eta\right),
$$

$$
\mathbf{v}=-\mathcal{L}_{c}^{-1} \nabla \cdot\left(1_{D} C G a+\eta\right)
$$


Let $\sigma:=1_{D} C G a+\eta$. Then, due to the minimizing property of $F_{C}(\sigma)$, we have the following identity

$$
\int_{\mathbb{R}^{d}} C F_{C}(\sigma): F_{C}(\sigma) d x=-\int_{\mathbb{R}^{d}} \sigma: F_{C}(\sigma) d x .
$$

Consequently, we have

$$
\begin{aligned}
& \min _{\mathbf{v} \in H^{1}\left(\mathbb{R}^{d}\right)} \int_{\mathbb{R}^{d}} C_{D}\left(\mathcal{E}(\mathbf{v})+1_{D} G a\right):\left(\mathcal{E}(\mathbf{v})+1_{D} G a\right) d x \\
& =\sup _{\eta \in L^{2}\left(\mathbb{R}^{d}: M_{d}^{S}\right)}\left[-\int_{\mathbb{R}^{d}}\left(C_{D}-C\right)^{-1} \eta: \eta d x+\int_{D} C G a: G a d x\right. \\
& \left.\quad+2 \int_{D} \eta: G a d x+\int_{\mathbb{R}^{d}} \sigma: F_{C}(\sigma)\right] \\
& =|D| a: G C\left(C-C^{1}\right)^{-1} C G a-|D| a: G C G a+\sup _{\sigma \in L^{2}\left(\mathbb{R}^{d}: M_{d}^{S}\right)} W_{a}(C, \sigma),
\end{aligned}
$$

where

$$
\begin{aligned}
W_{a}(C, \sigma) & =\int_{\mathbb{R}^{d}} \sigma: F_{C} \sigma+\int_{\mathbb{R}^{d}}\left(C-C_{D}\right)^{-1} \sigma: \sigma+2 \int_{D} \sigma:\left(C^{1}-C\right)^{-1} C^{1} G a \\
& =\int_{\mathbb{R}^{d}} \sigma: F_{C} \sigma+\int_{\mathbb{R}^{d}}\left(C-C_{D}\right)^{-1} \sigma: \sigma+2 \int_{D} \sigma:\left(C^{1}-C\right)^{-1}\left(C^{1}-C^{0}\right) a,
\end{aligned}
$$

as was given in (4.4). Since

$$
\begin{aligned}
& |D| a: G C\left(C-C^{1}\right)^{-1} C G a-|D| a: G C G a+|D|\left(C^{1}-C^{0}\right)\left(C^{1}\right)^{-1} C^{0} a: a \\
& =|D| a:\left(C^{1}-C^{0}\right)\left(C-C^{1}\right)^{-1}\left(C-C^{0}\right) a,
\end{aligned}
$$

we have proved (4.3).

The case when $C>C_{D}$ can be treated similarly. Writing $C_{D}=-C+C_{D}+C$, using the negativity of $C_{D}-C$, convex duality, and interchanging the order of minimization we obtain (4.5). This completes the proof.

We now derive trace bounds for EMT from identities (4.3) and (4.5). To state the bounds, let us fix some notation. Given a constant elasticity tensor $C=2 \mu_{c} \mathbf{I}_{4}+\lambda_{c} \mathbf{I}_{2} \otimes \mathbf{I}_{2}$, let $\mathcal{F}_{c}$ denote the symmetric positive definite tensors given by

$$
\mathcal{F}_{C}(\cdot)=\frac{1}{|D|} \int_{D} F_{C}\left(1_{D} \cdot\right) d x,
$$

where $F_{C}$ is defined by $(4.2)$, i.e.,

$$
\mathcal{F}_{C}(a)=\frac{1}{|D|} \int_{D} \mathcal{E L}_{c}^{-1} \nabla \cdot\left(1_{D} a\right) d x,
$$

and let $\mathcal{G}_{C}$ be the quadratic form given by

$$
\left\langle\mathcal{G}_{C} a, a\right\rangle=\frac{1}{|D|} \int_{\mathbb{R}^{d} \backslash D}\left(C^{1}-C^{0}\right) F_{C}\left(1_{D} a\right): F_{C}\left(1_{D} a\right) d x
$$

for $a \in M_{d}^{s}$. The main result of this paper is as follows. 
Theorem 4.2. Let $M$ be the EMT associated with the domain $D$, and $(\tilde{\lambda}, \tilde{\mu})$ and $(\lambda, \mu)$ be Lamé parameters of $D$ and the background, respectively. Then, we have

$$
\begin{aligned}
\frac{1}{|D|} \operatorname{tr}\left(\boldsymbol{\Lambda}_{1} M \boldsymbol{\Lambda}_{1}\right) \leq & d(\tilde{\kappa}-\kappa) \frac{d \kappa+2(d-1) \tilde{\mu}}{d \tilde{\kappa}+2(d-1) \tilde{\mu}}-d^{2}(\tilde{\kappa}-\kappa)^{2} \operatorname{tr}\left(\boldsymbol{\Lambda}_{1} \mathcal{G}_{C^{1}} \boldsymbol{\Lambda}_{1}\right) \\
\frac{1}{|D|} \operatorname{tr}\left(\boldsymbol{\Lambda}_{2} M \boldsymbol{\Lambda}_{2}\right) \leq & 2(\tilde{\mu}-\mu)\left[\frac{d^{2}+d-2}{2}-2(\tilde{\mu}-\mu)\left(\frac{d-2}{2 \tilde{\mu}}+\frac{d-1}{d \tilde{\kappa}+2(d-1) \tilde{\mu}}\right)\right] \\
& -d^{2}(\tilde{\kappa}-\kappa)^{2} \operatorname{tr}\left(\boldsymbol{\Lambda}_{2} \mathcal{G}_{C^{1}} \boldsymbol{\Lambda}_{2}\right)
\end{aligned}
$$

$$
|D| \operatorname{tr}\left(\boldsymbol{\Lambda}_{1} M^{-1} \boldsymbol{\Lambda}_{1}\right) \leq \frac{1}{d(\tilde{\kappa}-\kappa)} \frac{d \tilde{\kappa}+2(d-1) \mu}{d \kappa+2(d-1) \mu}
$$

$$
|D| \operatorname{tr}\left(\boldsymbol{\Lambda}_{2} M^{-1} \boldsymbol{\Lambda}_{2}\right) \leq \frac{1}{2(\tilde{\mu}-\mu)}\left[\frac{d^{2}+d-2}{2}+2(\tilde{\mu}-\mu)\left(\frac{d-2}{2 \mu}+\frac{d-1}{d \kappa+2(d-1) \mu}\right)\right] .
$$

Here $\operatorname{tr}$ denotes the trace and $\operatorname{tr}\left(\boldsymbol{\Lambda}_{k} \mathcal{G}_{C^{1}} \boldsymbol{\Lambda}_{k}\right), k=1,2$, is defined by

$$
\operatorname{tr}\left(\boldsymbol{\Lambda}_{k} \mathcal{G}_{C^{1}} \boldsymbol{\Lambda}_{k}\right)=\sum_{i, j=1}^{d}\left\langle\mathcal{G}_{C^{1}} \boldsymbol{\Lambda}_{k} E_{i j}, \boldsymbol{\Lambda}_{k} E_{i j}\right\rangle,
$$

where $E_{i j}:=\frac{1}{2}\left(\delta_{i k} \delta_{j l}+\delta_{i l} \delta_{j k}\right)_{k, l=1}^{d}$.

A few words on Theorem 4.2 are in order before proving it. We first note that if $C^{1}>C^{0}$ as we assumed, then $\left\langle\mathcal{G}_{C^{1}} \boldsymbol{\Lambda}_{k}, \boldsymbol{\Lambda}_{k}\right\rangle>0$ for $k=1,2$, and therefore inequalities (4.10) and (4.11) imply that

$$
\begin{aligned}
& \frac{1}{|D|} \operatorname{tr}\left(\boldsymbol{\Lambda}_{1} M \boldsymbol{\Lambda}_{1}\right)<d(\tilde{\kappa}-\kappa) \frac{d \kappa+2(d-1) \tilde{\mu}}{d \tilde{\kappa}+2(d-1) \tilde{\mu}} \\
& \frac{1}{|D|} \operatorname{tr}\left(\boldsymbol{\Lambda}_{2} M \boldsymbol{\Lambda}_{2}\right)<2(\tilde{\mu}-\mu)\left[\frac{d^{2}+d-2}{2}-2(\tilde{\mu}-\mu)\left(\frac{d-2}{2 \tilde{\mu}}+\frac{d-1}{d \tilde{\kappa}+2(d-1) \tilde{\mu}}\right)\right] .
\end{aligned}
$$

The bounds (4.12), (4.13), (4.15), and (4.16) are called Hashin-Shtrikman bounds for the EMT, and they were derived by Lipton [20]. The method of this paper yields those additional terms for the upper bounds and they will provide us with improved Hashin-Shtrikman bounds for thick domains (see the next section).

It is interesting to see whether the Hashin-Shtrikman bounds for EMTs are optimal or not. It is equally interesting to find a class of domains whose EMTs attain the lower bounds (4.12) and (4.13). For the PT the lower Hashin-Shtrikman bounds are attained by and only by ellipses and ellipsoids [16, 17]. The situation for the EMT is much more complicated. Since the EMT has $\frac{d(d+1)}{2}$ eigenvalues, those class must have $\frac{d(d+1)}{2}$ degree of freedom, and at this moment it is not clear what this class of domains must be. This question has an important application in the inverse problems to detect small elastic inclusions. For related work for the electrical impedance tomography, see $[3,5,9]$.

If $d=2$, then (4.12) and (4.13) read

$$
\begin{aligned}
& |D| \operatorname{tr}\left(\boldsymbol{\Lambda}_{1} M^{-1} \boldsymbol{\Lambda}_{1}\right) \leq \frac{\tilde{\kappa}+\mu}{2(\tilde{\kappa}-\kappa)(\kappa+\mu)}, \\
& |D| \operatorname{tr}\left(\boldsymbol{\Lambda}_{2} M^{-1} \boldsymbol{\Lambda}_{2}\right) \leq \frac{1}{\tilde{\mu}-\mu}+\frac{1}{2(\kappa+\mu)} .
\end{aligned}
$$


If $D$ is a two dimensional disk, it is proved in [5] that the corresponding EMT is isotropic and given by

$$
M=2|D| \frac{(\tilde{\kappa}-\kappa)(\kappa+\mu)}{\tilde{\kappa}+\mu} \boldsymbol{\Lambda}_{1}+2|D| \frac{2(\kappa+\mu)(\tilde{\mu}-\mu)}{2 \kappa+\mu+\tilde{\mu}} \boldsymbol{\Lambda}_{2} .
$$

Thus the equality in (4.12) and (4.13) are attained by a disk.

Proof of Theorem 4.2. Suppose that $C^{0}<C^{1}$. For $C<C^{0}$, using test functions of the form $\sigma=1_{D} b$, we obtain from (4.3),

$$
\begin{aligned}
a & :\left(\frac{1}{|D|} \widetilde{M}-\left(C^{1}-C^{0}\right)\left(C-C^{1}\right)^{-1}\left(C-C^{0}\right)\right) a \\
& +2 a:\left(C^{1}-C^{0}\right)\left(C-C^{1}\right)^{-1} b \geq\left(C-C^{1}\right)^{-1} b: b-b: \mathcal{F}_{C} b
\end{aligned}
$$

Because of (3.15) and (3.16), $\left(\frac{1}{|D|} \widetilde{M}-\left(C^{1}-C^{0}\right)\left(C-C^{1}\right)^{-1}\left(C-C^{0}\right)\right)$ is invertible on the subspace spanned by $\mathbf{I}_{2}$, or that of the trace-free matrices. Therefore, if either $a=\Lambda_{1} a$ or $a=\Lambda_{2} a$, we can choose $b$ so that

$$
a=-\left(\frac{1}{|D|} \widetilde{M}-\left(C^{1}-C^{0}\right)\left(C-C^{1}\right)^{-1}\left(C-C^{0}\right)\right)\left(C^{1}-C^{0}\right)\left(C-C^{1}\right)^{-1} b,
$$

and (4.17) becomes

$$
\begin{aligned}
& \left(\frac{1}{|D|} \widetilde{M}-\left(C^{1}-C^{0}\right)\left(C-C^{1}\right)^{-1}\left(C-C^{0}\right)\right)^{-1}\left(C^{1}-C^{0}\right)^{2}\left(C^{1}-C\right)^{-2} b: b \\
& \leq\left(C^{1}-C\right)^{-1} b: b+b: \mathcal{F}_{C} b .
\end{aligned}
$$

Taking the limit as $C$ tends to $C^{0}$ yields

$$
\left(\frac{1}{|D|} \widetilde{M}\right)^{-1} b: b \leq\left(C^{1}-C^{0}\right)^{-1} b: b+b: \mathcal{F}_{C^{0}} b,
$$

or equivalently,

$$
|D| \boldsymbol{\Lambda}_{j} M^{-1} \boldsymbol{\Lambda}_{j} \leq \boldsymbol{\Lambda}_{j}\left(C^{1}-C^{0}\right)^{-1} \boldsymbol{\Lambda}_{j}+\boldsymbol{\Lambda}_{j} \mathcal{F}_{C^{0}} \boldsymbol{\Lambda}_{j}, \quad j=1,2 .
$$

Therefore, we obtain

$$
\begin{aligned}
& |D| \operatorname{tr}\left(\boldsymbol{\Lambda}_{1} M^{-1} \boldsymbol{\Lambda}_{1}\right) \leq \frac{1}{d(\tilde{\kappa}-\kappa)} \operatorname{tr} \boldsymbol{\Lambda}_{1}+\operatorname{tr}\left(\boldsymbol{\Lambda}_{1} \mathcal{F}_{C^{0}} \boldsymbol{\Lambda}_{1}\right), \\
& |D| \operatorname{tr}\left(\boldsymbol{\Lambda}_{2} M^{-1} \boldsymbol{\Lambda}_{2}\right) \leq \frac{1}{2(\tilde{\mu}-\mu)} \operatorname{tr} \boldsymbol{\Lambda}_{2}+\operatorname{tr}\left(\boldsymbol{\Lambda}_{2} \mathcal{F}_{C^{0}} \boldsymbol{\Lambda}_{2}\right) .
\end{aligned}
$$

Since $\operatorname{tr} \boldsymbol{\Lambda}_{1}=1$ and $\operatorname{tr} \boldsymbol{\Lambda}_{2}=\frac{d(d+1)-2}{2},(4.20),(4.21)$, and Lemma 4.3 below lead us to (4.12) and (4.13).

We now prove (4.10)-(4.11). Let $U$ be an open subset of $\mathbb{R}^{d}$ disjoint with $D$. Given $\sigma_{D} \in L^{2}\left(\mathbb{R}^{d}: M_{d}^{S}\right)$ with the support in $D$ and given $C>C_{D}$, let $\sigma^{*}$ be given by

$$
\sigma^{*}=-C F_{C}\left(\sigma_{D}\right)
$$

and let

$$
\sigma_{U}=\mathbf{1}_{U}\left(C-C_{D}\right) C^{-1} \sigma^{*}
$$


Then one can easily see from (4.4) that

$$
\begin{aligned}
W_{a}\left(C, \sigma_{D}+\sigma_{U}\right) & =W_{a}\left(C, \sigma_{D}\right)+W_{a}\left(C, \sigma_{U}\right)+2 \int_{\mathbb{R}^{d}} \sigma_{U}: F_{C} \sigma_{D}, \\
& =W_{a}\left(C, \sigma_{D}\right)+\int_{\mathbb{R}^{d}} \sigma_{U}: F_{C} \sigma_{U}-\int_{U} C^{-1}\left(C-C_{D}\right) C^{-1} \sigma^{*}: \sigma^{*} .
\end{aligned}
$$

Since $F_{C}$ is negative definite, this yields that

$$
W_{a}(C, \sigma) \leq W_{a}\left(C, \sigma_{D}\right)-\int_{U} C^{-1}\left(C-C^{0}\right) C^{-1} \sigma^{*}: \sigma^{*} .
$$

If we use test functions of the form $\sigma_{D}=1_{D} b$ where $b \in M_{d}^{S}$, we obtain from (4.5), for $C>C^{1}$ that

$$
\begin{aligned}
\frac{1}{|D|} a: \widetilde{M} a \leq & \left(C^{1}-C^{0}\right)\left(C-C^{1}\right)^{-1}\left(C-C^{0}\right) a: a-2\left(C^{1}-C^{0}\right)\left(C-C^{1}\right)^{-1} a: b \\
& +\left(\left(C-C^{1}\right)^{-1}-\mathcal{F}_{C}\right) b: b-\frac{1}{|D|} \int_{U} C^{-1}\left(C-C^{0}\right) C^{-1} \sigma^{*}: \sigma^{*}
\end{aligned}
$$

where $\mathcal{F}_{c}$ is given by (4.8). If $C\left(>C^{1}\right)$ is sufficiently close to $C^{1}$, then $\left(\left(C-C^{1}\right)^{-1}-\mathcal{F}_{C}\right)$ is invertible, and hence we can choose

$$
\begin{aligned}
b & =\left(\left(C-C^{1}\right)^{-1}-\mathcal{F}_{C}\right)^{-1}\left(C^{1}-C^{0}\right)\left(C-C^{1}\right)^{-1} a \\
& =\left(\left(C^{1}-C^{0}\right)^{-1}-\left(C-C^{1}\right)\left(C^{1}-C^{0}\right)^{-1} \mathcal{F}_{C}\right)^{-1} a .
\end{aligned}
$$

Then the right hand side of (4.22) equals to

$$
a:\left(\mathbf{I}_{4}-\left(C-C^{0}\right) \mathcal{F}_{C}\right) b-\frac{1}{|D|} \int_{U} C^{-1}\left(C-C^{0}\right) C^{-1} \sigma^{*}: \sigma^{*}
$$

Taking the limit as $C$ tends to $C^{1}$ gives $b=\left(C^{1}-C^{0}\right) a$ and $\sigma^{*}=-C^{1} \mathcal{E}(\tilde{u})$, where $\tilde{u} \in H^{1}\left(\mathbb{R}^{d}: M_{d}^{S}\right)$ satisfies

$$
-\mathcal{L}_{C}(\tilde{u})=-\nabla \cdot\left(\mathbf{1}_{D}\left(C^{1}-C^{0}\right) a\right) .
$$

We have proved that

$$
\frac{1}{|D|} a: \widetilde{M} a \leq a:\left(\mathbf{I}_{4}-\left(C^{1}-C^{0}\right) \mathcal{F}_{C^{1}}\right)\left(C^{1}-C^{0}\right) a-\frac{1}{|D|} \int_{U}\left(C^{1}-C^{0}\right) \mathcal{E}(\tilde{u}): \mathcal{E}(\tilde{u}) .
$$

Since $U$ is arbitrary open subset of $\mathbb{R}^{d} \backslash D$, we have

$$
\frac{1}{|D|} a: \widetilde{M} a \leq a:\left(\mathbf{I}_{4}-\left(C^{1}-C^{0}\right) \mathcal{F}_{C^{1}}\right)\left(C^{1}-C^{0}\right) a-\left\langle\mathcal{G}_{C^{1}}\left(C^{1}-C^{0}\right) a,\left(C^{1}-C^{0}\right) a\right\rangle .
$$

So far we have shown that for $j=1,2$,

$$
\begin{aligned}
\frac{1}{|D|} \boldsymbol{\Lambda}_{j} M \boldsymbol{\Lambda}_{j} \leq & \boldsymbol{\Lambda}_{j}\left(C^{1}-C^{0}\right) \boldsymbol{\Lambda}_{j}-\boldsymbol{\Lambda}_{j}\left(C^{1}-C^{0}\right) \mathcal{F}_{C^{1}}\left(C^{1}-C^{0}\right) \boldsymbol{\Lambda}_{j} \\
& -\left\langle\mathcal{G}_{C^{1}}\left(C^{1}-C^{0}\right) \boldsymbol{\Lambda}_{j},\left(C^{1}-C^{0}\right) \boldsymbol{\Lambda}_{j}\right\rangle .
\end{aligned}
$$


Since $C^{1}$ and $C^{0}$ commute with $\boldsymbol{\Lambda}_{j}, j=1,2$, and $\boldsymbol{\Lambda}_{1}\left(C^{1}-C^{0}\right)=d(\tilde{\kappa}-\kappa) \boldsymbol{\Lambda}_{1}$ and $\boldsymbol{\Lambda}_{2}\left(C^{1}-\right.$ $\left.C^{0}\right)=2(\tilde{\mu}-\mu) \Lambda_{2}$, it follows from (4.23) that

$$
\begin{aligned}
\frac{1}{|D|} \operatorname{tr}\left(\boldsymbol{\Lambda}_{1} M \boldsymbol{\Lambda}_{1}\right) \leq & d(\tilde{\kappa}-\kappa)\left[\operatorname{tr} \boldsymbol{\Lambda}_{1}-d(\tilde{\kappa}-\kappa) \operatorname{tr}\left(\boldsymbol{\Lambda}_{1} \mathcal{F}_{C^{1}} \boldsymbol{\Lambda}_{1}\right)\right] \\
& -d^{2}(\tilde{\kappa}-\kappa)^{2} \operatorname{tr}\left(\boldsymbol{\Lambda}_{1} \mathcal{G}_{C^{1}} \boldsymbol{\Lambda}_{1}\right) \\
\frac{1}{|D|} \operatorname{tr}\left(\boldsymbol{\Lambda}_{2} M \boldsymbol{\Lambda}_{2}\right) \leq & 2(\tilde{\mu}-\mu)\left[\operatorname{tr} \boldsymbol{\Lambda}_{2}-2(\tilde{\mu}-\mu) \operatorname{tr}\left(\boldsymbol{\Lambda}_{2} \mathcal{F}_{C^{1}} \boldsymbol{\Lambda}_{2}\right)\right] \\
& -4(\tilde{\mu}-\mu)^{2} \operatorname{tr}\left(\boldsymbol{\Lambda}_{2} \mathcal{G}_{C^{1}} \boldsymbol{\Lambda}_{2}\right) .
\end{aligned}
$$

Here again, since $\operatorname{tr} \boldsymbol{\Lambda}_{1}=1$ and $\operatorname{tr} \boldsymbol{\Lambda}_{2}=\frac{d(d+1)-2}{2}$, we obtain the desired estimates from (4.24), (4.25), and Lemma 4.3 below. This completes the proof.

We now compute the trace of the symmetric operator $\mathcal{F}_{C}$ which has been used in the proof of Theorem 4.2. We include a detailed computation for the sake of completeness, (see [24] for example for another proof).

Lemma 4.3. Let $C=2 \mu_{c} \mathbf{I}_{4}+\lambda_{c} \mathbf{I}_{2} \otimes \mathbf{I}_{2}$. We have

$$
\begin{aligned}
\operatorname{tr}\left(\mathcal{F}_{C}\right) & =\left(\frac{1}{\lambda_{c}+2 \mu_{c}}+\frac{d-2}{2 \mu_{c}}\right)|D|, \\
\operatorname{tr}\left(\boldsymbol{\Lambda}_{1} \mathcal{F}_{C} \boldsymbol{\Lambda}_{1}\right) & =\frac{1}{d\left(\lambda_{c}+2 \mu_{c}\right)}|D|, \\
\operatorname{tr}\left(\boldsymbol{\Lambda}_{2} \mathcal{F}_{C} \boldsymbol{\Lambda}_{2}\right) & =\left(\frac{d-1}{d\left(\lambda_{c}+2 \mu_{c}\right)}+\frac{d-2}{2 \mu_{c}}\right)|D| .
\end{aligned}
$$

Proof. By taking the Fourier transform, we obtain

$$
\widehat{\mathcal{L}_{C} \mathbf{u}}(\xi)=-\left(\mu_{c}|\xi|^{2} \mathbf{I}_{2}+\left(\lambda_{c}+\mu_{c}\right)(\xi \otimes \xi)\right) \hat{\mathbf{u}}(\xi) .
$$

Let $\xi^{1}:=\xi /|\xi|$ and $\xi^{j}, j=2 \ldots d$, be unit vectors such that $\left\{\xi^{1}, \ldots, \xi^{d}\right\}$ forms an orthonormal basis for $\mathbb{R}^{d}$. Then, we have

$$
\mathbf{I}_{2}=\sum_{j=1}^{d} \xi^{j} \otimes \xi^{j}
$$

It then follows that

$$
\widehat{\mathcal{L}_{C} \mathbf{u}}(\xi)=-|\xi|^{2}\left[\left(\lambda_{c}+2 \mu_{c}\right) \xi^{1} \otimes \xi^{1}+\mu_{c} \sum_{j=2}^{d} \xi^{j} \otimes \xi^{j}\right] \hat{\mathbf{u}}(\xi),
$$

and hence

$$
\begin{aligned}
\widehat{\mathcal{L}_{C}^{-1} \mathbf{u}}(\xi) & =-\frac{1}{|\xi|^{2}}\left[\frac{1}{\lambda_{c}+2 \mu_{c}} \xi^{1} \otimes \xi^{1}+\frac{1}{\mu_{c}} \sum_{j=2}^{d} \xi^{j} \otimes \xi^{j}\right] \hat{\mathbf{u}}(\xi) \\
& =-\frac{1}{|\xi|^{4}}\left[\left(\frac{1}{\lambda_{c}+2 \mu_{c}}-\frac{1}{\mu_{c}}\right) \xi \otimes \xi+\frac{|\xi|^{2}}{\mu_{c}} \mathbf{I}_{2}\right] \hat{\mathbf{u}}(\xi) .
\end{aligned}
$$


Let $a$ be a $d \times d$ symmetric matrix. Then,

$$
\begin{aligned}
\mathcal{E}\left(\mathcal{L}_{C}^{-1} \nabla \cdot\left(1_{D} a\right)\right)(\xi)= & \left.\left.-\frac{i}{2}\left[\xi \otimes \mathcal{L}_{C}^{-1} \widehat{\nabla \cdot\left(1_{D}\right.} a\right)+\mathcal{L}_{C}^{-1} \widehat{\nabla \cdot\left(1_{D}\right.} a\right) \otimes \xi\right] \\
= & \frac{1}{2|\xi|^{4}}\left[\xi \otimes\left(\left(\frac{1}{\lambda_{c}+2 \mu_{c}}-\frac{1}{\mu_{c}}\right) \xi \otimes \xi+\frac{|\xi|^{2}}{\mu_{c}} \mathbf{I}_{2}\right) a \xi \widehat{1_{D}}\right. \\
& \left.+\left(\left(\left(\frac{1}{\lambda_{c}+2 \mu_{c}}-\frac{1}{\mu_{c}}\right) \xi \otimes \xi+\frac{|\xi|^{2}}{\mu_{c}} \mathbf{I}_{2}\right) a \xi \widehat{1_{D}}\right) \otimes \xi\right] .
\end{aligned}
$$

Since $a$ is symmetric, we then get

$$
\begin{aligned}
\left.1_{D} a: \mathcal{E}\left(\mathcal{L}_{C}^{-\widehat{1} \nabla \cdot\left(1_{D}\right.} a\right)\right) & =\left|\widehat{1_{D}}\right|^{2} a: \xi \otimes\left[\left(\frac{1}{\lambda_{c}+2 \mu_{c}}-\frac{1}{\mu_{c}}\right) \frac{\xi \otimes \xi}{|\xi|^{4}}+\frac{1}{\mu_{c}|\xi|^{2}} \mathbf{I}_{2}\right] a \xi \\
& =\left|\widehat{1_{D}}\right|^{2}\left[\left(\frac{1}{\lambda_{c}+2 \mu_{c}}-\frac{1}{\mu_{c}}\right) \frac{(a \xi \cdot \xi)^{2}}{|\xi|^{4}}+\frac{|a \xi|^{2}}{\mu_{c}|\xi|^{2}}\right] .
\end{aligned}
$$

We now have

$$
\begin{aligned}
& \left.\int_{\mathbb{R}^{d}} 1_{D} a: \mathcal{F}_{C}\left(1_{D} a\right) d x=\frac{1}{(2 \pi)^{d}} \int_{\mathbb{R}^{d}} \widehat{1_{D}} a: \widehat{\mathcal{F}_{C}\left(1_{D} a\right.}\right) d \xi \\
& =\frac{1}{(2 \pi)^{d}}\left(\frac{1}{\lambda_{c}+2 \mu_{c}}-\frac{1}{\mu_{c}}\right) \int_{\mathbb{R}^{d}}\left|\widehat{1_{D}}\right|^{2} \frac{(a \xi \cdot \xi)^{2}}{|\xi|^{4}} d \xi+\frac{1}{(2 \pi)^{d} \mu_{c}} \int_{\mathbb{R}^{d}}\left|\widehat{1_{D}}\right|^{2} \frac{|a \xi|^{2}}{|\xi|^{2}} d \xi .
\end{aligned}
$$

Then

$$
\begin{aligned}
\operatorname{tr}\left(\mathcal{F}_{C}\right)= & \sum_{i, j=1}^{d} \int_{\mathbb{R}^{d}} 1_{D} E_{i j}: \mathcal{F}_{C}\left(1_{D} E_{i j}\right) d x \\
= & \frac{1}{(2 \pi)^{d}}\left(\frac{1}{\lambda_{c}+2 \mu_{c}}-\frac{1}{\mu_{c}}\right) \sum_{i, j=1}^{d} \int_{\mathbb{R}^{d}}\left|\widehat{1_{D}}\right|^{2} \frac{\left(\xi_{i} \xi_{j}\right)^{2}}{|\xi|^{4}} d \xi \\
& +\frac{1}{(2 \pi)^{d} \mu_{c}} \sum_{i, j=1}^{d} \int_{\mathbb{R}^{d}}\left|\widehat{1_{D}}\right|^{2} \frac{\left(\xi_{i}^{2}+\xi_{j}^{2}\right)}{4|\xi|^{2}} d \xi \\
= & \left(\frac{1}{\lambda_{c}+2 \mu_{c}}-\frac{1}{\mu_{c}}\right)|D|+\frac{d}{2 \mu_{c}}|D| \\
= & \left(\frac{1}{\lambda_{c}+2 \mu_{c}}+\frac{d-2}{2 \mu_{c}}\right)|D| .
\end{aligned}
$$

To prove (4.27), we apply the above formula to $a=\mathbf{I}_{2}$, and (4.28) follows from (4.26) and (4.27). This completes the proof.

\section{Improved Hashin-Shtrikman Bounds for Thick Domains}

We now estimate the trace of $\mathcal{G}_{C}$, in particular, the bulk part $\operatorname{tr}\left(\boldsymbol{\Lambda}_{1} \mathcal{G}_{C} \boldsymbol{\Lambda}_{1}\right)$ for thick domains. We first have the following lemma.

Lemma 5.1. Let $C=2 \mu_{c} \mathbf{I}_{4}+\lambda_{c} \mathbf{I}_{2} \otimes \mathbf{I}_{2}$. We have

$$
\operatorname{tr}\left(\boldsymbol{\Lambda}_{1} \mathcal{G}_{C} \boldsymbol{\Lambda}_{1}\right)=2(\tilde{\mu}-\mu)\left(\frac{1}{\omega_{d}\left(2 \mu_{c}+\lambda_{c}\right)}\right)^{2} \mathcal{K}(D),
$$


where

$$
\mathcal{K}(D)=\frac{1}{|D|} \sum_{i, j=1}^{d} \int_{\mathbb{R}^{d} \backslash D}\left(\int_{D}\left(\frac{1}{d} \delta_{i j}-\frac{\left(x_{i}-y_{i}\right)\left(x_{j}-y_{j}\right)}{|x-y|^{2}}\right) \frac{d y}{|x-y|^{d}}\right)^{2} d x
$$

Proof. For a given constant symmetric matrix $a, F_{C}\left(1_{D} a\right)$ can be written in terms of the fundamental solution $\Gamma$ of the Lamé system $\mathcal{L}_{C}$. In fact, let $\Phi \in H^{1}\left(\mathbb{R}^{d}: M_{d}^{S}\right)$ be the solution of

$$
\mathcal{L}_{C}(\Phi)=\nabla \cdot\left(1_{D} a\right)
$$

Then, for all $i \in\{1, \ldots, d\}$,

$$
\begin{aligned}
\Phi_{i}(x) & =\sum_{j=1}^{d} \int_{\mathbb{R}^{d}} \Gamma_{i j}(x-y)\left[\sum_{k=1}^{d} \frac{\partial}{\partial y_{k}}\left(1_{D}(y) a_{j k}\right)\right] d y \\
& =-\sum_{j, k=1}^{d} \int_{\mathbb{R}^{d}} \frac{\partial}{\partial y_{k}} \Gamma_{i j}(x-y) 1_{D}(y) a_{j k} d y \\
& =\sum_{j, k=1}^{d} \frac{\partial}{\partial x_{k}}\left(\int_{\mathbb{R}^{d}} \Gamma_{i j}(x-y) 1_{D}(y) a_{j k} d y\right) .
\end{aligned}
$$

If we define

$$
\varphi(x)=\int_{D} \boldsymbol{\Gamma}(x-y) d y
$$

we have $\Phi_{i}=\sum_{j, k} \frac{\partial}{\partial x_{k}}\left(\varphi_{i j} a_{j k}\right)$, and

$$
\left(F_{C}\left(1_{D} a\right)\right)_{i p}=\sum_{j, k} \mathcal{E}_{j k}^{i p}(\varphi) a_{j k}
$$

where

$$
\mathcal{E}_{j k}^{i p}(\varphi)=\frac{1}{2} \frac{\partial \varphi_{i j}}{\partial x_{p} \partial x_{k}}+\frac{1}{2} \frac{\partial \varphi_{p j}}{\partial x_{i} \partial x_{k}}
$$

Let $\omega_{d}$ be the surface area of the unit sphere in $\mathbb{R}^{d}$, i.e., $\omega_{2}=2 \pi$ and $\omega_{3}=4 \pi$. Then, we have

$$
\begin{aligned}
\omega_{d}|x|^{d} \frac{\partial^{2}}{\partial x_{k} \partial x_{l}} \Gamma_{i j}(x)= & A \delta_{i j}\left(\delta_{k l}-d \frac{x_{k} x_{l}}{|x|^{2}}\right)-B\left(\delta_{i k} \delta_{l j}+\delta_{j k} \delta_{i l}\right) \\
& +\frac{d B}{|x|^{2}}\left(\delta_{i k} x_{j} x_{l}+\delta_{j k} x_{i} x_{l}+\delta_{i l} x_{j} x_{k}+\delta_{j l} x_{i} x_{k}+\delta_{k l} x_{i} x_{j}\right) \\
& -\frac{d(d+2) B}{|x|^{4}} x_{i} x_{j} x_{k} x_{l} .
\end{aligned}
$$


In particular, if $j=l$, the equation simplifies into

$$
\begin{aligned}
\omega_{d}|x|^{d} \frac{\partial^{2}}{\partial x_{k} \partial x_{j}} \Gamma_{i j}(x)= & A \delta_{i j}\left(\delta_{i k}-d \frac{x_{k} x_{i}}{|x|^{2}}\right)-B \delta_{i k}\left(1+\delta_{j i}\right) \\
& +d B \frac{x_{i} x_{k}}{|x|^{2}}\left(2 \delta_{j k}+\delta_{i j}+1\right) \\
& +d B\left(\delta_{i k}-(d+2) \frac{x_{i} x_{k}}{|x|^{2}}\right) \frac{x_{j}^{2}}{|x|^{2}} .
\end{aligned}
$$

Consequently, we obtain

$$
\sum_{j=1}^{d} \frac{\partial \Gamma_{i j}}{\partial x_{k} \partial x_{j}}=\frac{1}{|x|^{d}} \frac{(A-B)}{\omega_{d}}\left(\delta_{i k}-d \frac{x_{k} x_{i}}{|x|^{2}}\right)=\frac{1}{|x|^{d}} \frac{1}{\omega_{d}\left(2 \mu_{c}+\lambda_{c}\right)}\left(\delta_{i k}-d \frac{x_{k} x_{i}}{|x|^{2}}\right) .
$$

Let $\theta(x)=\frac{x}{|x|}$ for $x \neq 0$. Since $\boldsymbol{\Lambda}_{\mathbf{1}}(a)=\frac{\operatorname{tr}(a)}{d} \mathbf{I}_{2}$, we have

$$
\begin{aligned}
F_{C}\left(1_{D} \boldsymbol{\Lambda}_{1}(a)\right)_{i j}(x) & =\frac{\operatorname{tr}(a)}{d} \sum_{k=1}^{d} \mathcal{E}_{i j}^{k k}(\varphi) \\
& =\frac{\operatorname{tr}(a)}{d \omega_{d}\left(2 \mu_{c}+\lambda_{c}\right)} \int_{D}\left(\delta_{i j}-d \theta_{i}(x-y) \theta_{j}(x-y)\right) \frac{d y}{|x-y|^{d}} .
\end{aligned}
$$

This last formula can be written as

$$
F_{C}\left(1_{D} \boldsymbol{\Lambda}_{1}(a)\right)(x)=\frac{\operatorname{tr}(a)}{\omega_{d}\left(2 \mu_{c}+\lambda_{c}\right)}\left(\frac{1}{d} \int_{D} \frac{d y}{|x-y|^{d}} \mathbf{I}_{2}-\int_{D} \theta(x-y) \otimes \theta(x-y) \frac{d y}{|x-y|^{d}}\right) .
$$

Note that $F_{C}\left(1_{D} \boldsymbol{\Lambda}_{1}(a)\right)(x)$ is trace free, in other words,

$$
\left(\mathbf{I}_{2} \otimes \mathbf{I}_{2}\right) F_{C}\left(1_{D} \boldsymbol{\Lambda}_{1}(a)\right)=0 .
$$

Consequently, we have

$$
\left(C^{1}-C^{0}\right) F_{C}\left(1_{D} \boldsymbol{\Lambda}_{1}(a)\right)(x)=2(\tilde{\mu}-\mu) F_{C}\left(1_{D} \boldsymbol{\Lambda}_{1}(a)\right)(x)
$$

since $C^{1}-C^{0}=(\tilde{\lambda}-\lambda) \mathbf{I}_{2} \otimes \mathbf{I}_{2}+2(\tilde{\mu}-\mu) \mathbf{I}_{4}$.

The computation of $\left\langle\mathcal{G}_{C} \boldsymbol{\Lambda}_{1}(a), \boldsymbol{\Lambda}_{1}(a)\right\rangle$ is now straightforward. If we set

$$
C=2(\tilde{\mu}-\mu)\left(\frac{1}{\omega_{d}\left(2 \mu_{c}+\lambda_{c}\right)}\right)^{2},
$$

then

$$
\begin{aligned}
& \left\langle\mathcal{G}_{C} \boldsymbol{\Lambda}_{1}(a), \boldsymbol{\Lambda}_{1}(a)\right\rangle \\
& =\frac{1}{|D|} \int_{\mathbb{R}^{d} \backslash D}\left(C^{1}-C^{0}\right) F_{C}\left(1_{D} \boldsymbol{\Lambda}_{1}(a)\right): F_{C}\left(1_{D} \boldsymbol{\Lambda}_{1}(a)\right) \\
& =\frac{C \operatorname{tr}(a)^{2}}{|D|} \sum_{i, j=1}^{d} \int_{\mathbb{R}^{d} \backslash D}\left(\int_{D}\left(\frac{1}{d} \delta_{i j}-\theta_{i}(x-y) \theta_{j}(x-y)\right) \frac{d y}{|x-y|^{d}}\right)^{2} d x,
\end{aligned}
$$

which in turn yields formula (5.1). 
We now derive a lower bound for $\mathcal{K}(D)$ depending on shape of $D$. The characteristic feature of the shape that we shall use is its thickness. Suppose that $D$ is of given thickness $\tau$ as defined in (1.5). Then $D$ is contained in $B_{r}\left(x_{0}\right)$ for some $x_{0}$ where

$$
r=\left(\frac{d|D|}{\tau \omega_{d}}\right)^{\frac{1}{d}}
$$

One can easily see that

$$
\begin{aligned}
|D| \mathcal{K}(D) & =\sum_{i, j=1}^{d} \int_{\mathbb{R}^{d} \backslash D}\left(\int_{D}\left(\frac{1}{d} \delta_{i j}-\theta_{i}(x-y) \theta_{j}(x-y)\right) \frac{d y}{|x-y|^{d}}\right)^{2} d x \\
& =\int_{\mathbb{R}^{d} \backslash D}\left[\sum_{i, j=1}^{d}\left(\int_{D} \theta_{i}(x-y) \theta_{j}(x-y) \frac{d y}{|x-y|^{d}}\right)^{2}-\frac{1}{d}\left(\int_{D} \frac{d y}{|x-y|^{d}}\right)^{2}\right] d x .
\end{aligned}
$$

A simple computation shows that the integrand of the above integral in $x$ is non-negative. Indeed, if we define

$$
X_{i}:=\int_{D} \theta_{i}^{2}(x-y) \frac{d y}{|x-y|^{d}}, \quad i=1, \ldots, d,
$$

then

$$
\sum_{i=1}^{d} X_{i}=\int_{D} \frac{d y}{|x-y|^{d}}
$$

and we have immediately

$$
\sum_{i=1}^{d}\left(\int_{D} \theta_{i}^{2}(x-y) \frac{d y}{|x-y|^{d}}\right)^{2} \geq \frac{1}{d}\left(\int_{D} \frac{d y}{|x-y|^{d}}\right)^{2} .
$$

Therefore, a lower bound on $\mathcal{K}(D)$ is obtained by reducing the domain of integration, i.e., (5.6)

$$
|D| \mathcal{K}(D) \geq \int_{\mathbb{R}^{d} \backslash B_{t r}\left(x_{0}\right)}\left[\sum_{i, j=1}^{d}\left(\int_{D} \theta_{i}(x-y) \theta_{j}(x-y) \frac{d y}{|x-y|^{d}}\right)^{2}-\frac{1}{d}\left(\int_{D} \frac{d y}{|x-y|^{d}}\right)^{2}\right] d x
$$

where $t>1$ is a parameter to be chosen later. Note that

$$
\begin{aligned}
& \sum_{i, j=1}^{d}\left(\int_{D} \theta_{i}(x-y) \theta_{j}(x-y) \frac{d y}{|x-y|^{d}}\right)^{2} \\
& =\sum_{i, j=1}^{d} \int_{D \times D} \theta_{i}(x-y) \theta_{j}(x-y) \theta_{i}\left(x-y^{\prime}\right) \theta_{j}\left(x-y^{\prime}\right) \frac{d y d y^{\prime}}{|x-y|^{d}\left|x-y^{\prime}\right|^{d}} \\
& =\int_{D \times D} \frac{\left\langle x-y, x-y^{\prime}\right\rangle^{2}}{|x-y|^{d+2}\left|x-y^{\prime}\right|^{d+2}} d y d y^{\prime} .
\end{aligned}
$$

Since $D \subset B_{r}\left(x_{0}\right)$, we have

$$
\left\langle x-y, x-y^{\prime}\right\rangle \geq \frac{t^{2}-1}{t^{2}+1}|x-y|\left|x-y^{\prime}\right|
$$


for all $x \in \mathbb{R}^{d} \backslash B_{t r}\left(x_{0}\right)$ and $y, y^{\prime} \in D$, and consequently

$$
\sum_{i, j=1}^{d}\left(\int_{D} \theta_{i}(x-y) \theta_{j}(x-y) \frac{d y}{|x-y|^{d}}\right)^{2} \geq\left(\frac{t^{2}-1}{t^{2}+1}\right)^{2}\left(\int_{D} \frac{d y}{|x-y|^{d}}\right)^{2} .
$$

We have obtained that

$$
|D| \mathcal{K}(D) \geq\left(\frac{t^{2}-1}{t^{2}+1}\right)^{2} \int_{\mathbb{R}^{d} \backslash B_{t r}\left(x_{0}\right)}\left(\int_{D} \frac{d y}{|x-y|^{d}}\right)^{2} d x .
$$

Now remark that

$$
\frac{1}{|x-y|} \geq \frac{t}{t+1} \frac{1}{\left|x-x_{0}\right|} \text { for all } x \in \mathbb{R}^{d} \backslash B_{t r}\left(x_{0}\right) \text { and } y \in B_{r}\left(x_{0}\right) .
$$

It then follows from (5.7) and (5.5) that

$$
\mathcal{K}(D) \geq \frac{\left(t^{2}-1\right)^{2} t^{d}}{\left(t^{2}+1\right)^{2}(t+1)^{2 d}}\left(\frac{\omega_{d}}{d}\right)^{2} \tau
$$

We now choose $t$ to optimize estimate (5.9), and set

$$
C_{d}:=\max _{t>1} \frac{\left(t^{2}-1\right)^{2} t^{d}}{\left(t^{2}+1\right)^{2}(t+1)^{2 d}}
$$

Inserting this result in (4.10) and (5.1) we have proved the following proposition.

Proposition 5.2. Suppose that the thickness of $D$ is $\tau$. Then

$$
\frac{1}{|D|} \operatorname{tr}\left(\boldsymbol{\Lambda}_{1} M \boldsymbol{\Lambda}_{1}\right) \leq d(\tilde{\kappa}-\kappa) \frac{d \kappa+2(d-1) \tilde{\mu}}{d \tilde{\kappa}+2(d-1) \tilde{\mu}}-\delta \tau,
$$

where

$$
\delta:=C_{d} d^{2}(\tilde{\mu}-\mu)\left(\frac{\tilde{\kappa}-\kappa}{d \tilde{\kappa}+2(d-1) \tilde{\mu}}\right)^{2} .
$$

\section{Size Estimation for an InClusion}

In this section we discuss one important application of the Hashin-Shtrikman bounds. Suppose that an elastic body occupies a bounded domain $\Omega$ in $\mathbb{R}^{d}$ whose boundary $\partial \Omega$ is Lipschitz. Suppose that $\Omega$ contains an inclusion $D$ of small volume. Assume that $D$ is of the form

$$
D=\epsilon B+z,
$$

where the small constant $\epsilon$ represents the smallness of the volume of $D, B$ is a reference domain, and $z$ represents the location of the inclusion. Suppose that the background $\Omega$ has the isotropic elasticity tensor $C^{0}=2 \mu \mathbf{I}_{4}+\lambda \mathbf{I}_{2} \otimes \mathbf{I}_{2}$, while that of the inclusion is $C^{1}=2 \tilde{\mu} \mathbf{I}_{4}+\tilde{\lambda} \mathbf{I}_{2} \otimes \mathbf{I}_{2}$. We assume that either $C^{1}>C^{0}$ or $C^{1}<C^{0}$. Then the elasticity tensor for $\Omega$ with the inclusion is given by

$$
C=1_{\Omega \backslash D} C^{0}+1_{D} C^{1} .
$$

We consider an inverse problem to identify the unknown inclusion $D$ by the tractiondisplacement measured on $\partial \Omega$. 
Given a traction $\mathbf{g}$ on $\partial \Omega$, the displacement $\mathbf{u}_{\epsilon}$ is the solution to

$$
\begin{cases}\nabla \cdot\left(C \mathcal{E}\left(\mathbf{u}_{\epsilon}\right)\right)=0 & \text { in } \Omega, \\ \frac{\partial \mathbf{u}_{\epsilon}}{\partial \nu}=\mathbf{g} & \text { on } \partial \Omega .\end{cases}
$$

Let $\mathbf{u}$ be the solution without the inclusion, i.e., $\nabla \cdot\left(C^{0} \mathcal{E}(\mathbf{u})\right)=0$ in $\Omega$ and $\frac{\partial \mathbf{u}}{\partial \nu}=\mathbf{g}$ on $\partial \Omega$. Then it is proved in [7] that if $\mathbf{g}=\frac{\partial \mathbf{u}}{\partial \nu}$ for a linear displacement $\mathbf{u}=a x$ for some $d \times d$ symmetric matrix $a$, then

$$
\mathbf{u}_{\epsilon}(x)-\mathbf{u}(x)+\sum_{i, j, p, q=1}^{d} a_{i j} \partial_{p} N_{k q}(x-z) m_{i j p q}+O\left(\epsilon^{2 d}\right), \quad x \in \partial \Omega .,
$$

where $M=\left(m_{i j p q}\right)$ is the EMT associated with the inclusion $D$ and $N$ is the Neumann function for $\mathcal{L}_{C}$ on $\Omega$. It then follows that if $b$ is another $d \times d$ symmetric matrix, then

$$
\int_{\partial \Omega}\left[\mathbf{u}_{\epsilon}(x)-\mathbf{u}(x)\right] \cdot \frac{\partial(b x)}{\partial \nu} d \sigma=a: M b+O\left(\epsilon^{2 d}\right)
$$

The formula (6.3) says that the EMT $M$ associated with the single inclusion $D$ can be detected from the boundary measurement $\mathbf{u}_{\epsilon}$ on $\partial \Omega$. We then obtain the following theorem from Theorem 4.2 and Proposition 5.2.

Theorem 6.1. Let $M$ be the EMT detected from the boundary measurement via the formula (6.3) and let $\kappa_{1}, \kappa_{2}, \kappa_{3}, \kappa_{4}$ be the constants on the right hand sides of (4.10)-(4.12) consecutively. Then

$$
\max \left(\frac{\operatorname{tr}\left(\boldsymbol{\Lambda}_{1} M \boldsymbol{\Lambda}_{1}\right)}{\kappa_{1}}, \frac{\operatorname{tr}\left(\boldsymbol{\Lambda}_{2} M \boldsymbol{\Lambda}_{2}\right)}{\kappa_{2}}\right) \leq|D| \leq \min \left(\frac{\kappa_{3}}{\operatorname{tr}\left(\boldsymbol{\Lambda}_{1} M^{-1} \boldsymbol{\Lambda}_{1}\right)}, \frac{\kappa_{4}}{\operatorname{tr}\left(\boldsymbol{\Lambda}_{2} M^{-1} \boldsymbol{\Lambda}_{2}\right)}\right)
$$

provided that $C^{1}>C^{0}$. If we know a priori an upper bound of the thickness of $D$, say $r$, then we have

$$
\frac{\operatorname{tr}\left(\boldsymbol{\Lambda}_{1} M \boldsymbol{\Lambda}_{1}\right)}{\kappa_{1}-\delta \tau} \leq|D| \leq \min \left(\frac{\kappa_{3}}{\operatorname{tr}\left(\boldsymbol{\Lambda}_{1} M^{-1} \boldsymbol{\Lambda}_{1}\right)}, \frac{\kappa_{4}}{\operatorname{tr}\left(\boldsymbol{\Lambda}_{2} M^{-1} \boldsymbol{\Lambda}_{2}\right)}\right)
$$

where $\delta$ is defined in Proposition 5.2. If $C^{1}<C^{0}$, then inequalities are reversed.

We note that (6.4) and (6.5) are improved estimates of the size of $D$ over those in $[7,15]$.

Acknowledgement. We thank Graeme W. Milton for pointing out to us the existence of Lipton's work [20].

\section{REFERENCES}

[1] H. Ammari, Y. Capdeboscq, H. Kang, E. Kim, and M. Lim, Attainability by Simply Connected Domains of Optimal Bounds for Polarization Tensors, European J. Applied Math. 17(2) (2006), 201-219.

[2] H. Ammari and H. Kang, High-order terms in the asymptotic expansions of the steady-state voltage potentials in the presence of conductivity inhomogeneities of small diameter. SIAM J. Math. Anal., $34(5)$ (2003), 1152-1166.

[3] ——-, Reconstruction of Small Inhomogeneities from Boundary Measurements, Lecture Notes in Mathematics, 1846, Springer, 2004.

[4] - - Polarization and Moment Tensors with Applications to Inverse Problems and Effective Medium Theory, Springer-Verlag, to appear.

[5] H. Ammari, H. Kang, E. Kim, and M. Lim, Reconstruction of closely spaced small inclusions. SIAM J. Numer. Anal., 42(6) (2005), 2408-2428. 
[6] H. Ammari, H. Kang, and M. Lim, Effective Parameters of Elastic Composites, Indiana Univ. Math. J., 55(3) (2006), 903-922.

[7] H. Ammari, H. Kang, G. Nakamura, and K. Tanuma, Complete asymptotic expansions of solutions of the system of elastostatics in the presence of an inclusion of small diameter and detection of an inclusion. J. Elasticity, 67(2) (2002), 97-129.

[8] H. Ammari, H. Kang, and K. Touibi, Boundary layer techniques for deriving the effective properties of composite materials. Asymptot. Anal., 41(2) (2005), 119-140.

[9] M. Brühl, M. Hanke, and M. S. Vogelius, A direct impedance tomography algorithm for locating small inhomogeneities. Numer. Math., 93(4) (2003), 635-654.

[10] Y. Capdeboscq and H. Kang, Improved Bounds on the Polarization Tensor for Thick Domains, in Inverse Problems, Multi-scale Analysis and Effective Medium Theory, Contemp. Math., 408, 69-74, Amer. Math. Soc., 2006.

[11] Y. Capdeboscq and M. S. Vogelius, A general representation formula for boundary voltage perturbations caused by internal conductivity inhomogeneities of low volume fraction. M2AN Math. Model. Numer. Anal., 37(1) (2003), 159-173.

[12] Y. Capdeboscq and M. S. Vogelius, A review of some recent work on impedance imaging for inhomogeneities of low volume fraction, in Partial differential equations and inverse problems, Contemp. Math. 362, 69-87, Amer. Math. Soc., 2004.

[13] A. Friedman and M. Vogelius, Identification of small inhomogeneities of extreme conductivity by boundary measurements: a theorem on continuous dependence, Arch. Rat. Mech. Anal. 105 (1989), 299-326.

[14] Z. Hashin and S. Shtrikman, A variational approach to the theory of the elastic behavior of multiphase materials, J.Mech. Phys. Solids, 11 (1963), 127-140.

[15] H. Kang, E. Kim, and J. Lee, Identification of Elastic Inclusions and Elastic Moment Tensors by Boundary Measurements, Inverse Problems 19 (2003), 703-724.

[16] H. Kang and G.W. Milton, On Conjectures of Polya-Szego and Eshelby, in Inverse Problems, Multi-scale Analysis and Effective Medium Theory, Contemp. Math., 408, 75-80, Amer. Math. Soc. 2006.

[17] —— Solutions to the Conjectures of Pólya-Szegö and Eshelby, submitted.

[18] R. V. Kohn and G. W. Milton, On bounding the effective conductivity of anisotropic composites. In Homogenization and effective moduli of materials and media (Minneapolis, Minn., 1984/1985), IMA Vol. Math. Appl., 1, 97-125. Springer, 1986.

[19] Energy change due to the appearance of cavity in solids. Int. J. Solids Struct., 40(7) (1993), 1765-1803.

[20] R. Lipton, Inequalities for electric and elastic polarization tensors with applications to random composites. J. Mech. Phys. Solids, 41(5) (1993), 809-833.

[21] K.A. Lurie and A.V. Cherkaev, G-closure of a set of anisotropic conducting media in the case of twodimensions, Dokl. Akad. Nauk SSSR, 259 (1981), 328-331, translated in Soviet Phys. Dokl., 26 (1981), 657-659.

[22] K.A. Lurie and A.V. Cherkaev, Exact estimates of the conductivity of a binary mixture of isotropic materials, Proc. Roy. Soc. Edinburgh A 104 (1986), 21-38.

[23] V. G. Maz'ya and S. A. Nazarov, Asymptotic behavior of energy integrals under small perturbations of the boundary near corner and conic points. Trudy Moskov. Mat. Obshch., 50 (1987), 79-129.

[24] G. W. Milton, The Theory of Composites. Cambridge Monographs on Applied and Computational Mathematics. Cambridge University Press, 2002.

[25] G.W. Milton, R.C. McPhedran, and D.R. McKenzie, Transport properties of arrays of intersecting cylinders, Appl. Phys. 25 (1981) 23-30.

[26] A. B. Movchan and S. K. Serkov, The Pólya-Szegó matrices in asymptotic models of dilute composites. European J. Appl. Math., 8(6) (1997), 595-621.

[27] F. Murat and L. Tartar, Calcul des variations et homogénísation. In Les méthodes de l'homogénéisation: théorie et applications en physique Collection de la Direction des études et recherches d'Électricité de France, Eyrolles, Paris, 57 (1985) 319-370. [Translated in Topics in the Mathematical Modeling of Composite Materials, ed. by A. Cherkaev and R. Kohn., Progress in Nonlinear Differential Equations and Their Applications Volume 31, pp. 139-173, Birkhäuser.]

[28] G. Pólya and G. Szegö, Isoperimetric Inequalities in Mathematical Physics. Annals of Mathematics Studies, no. 27. Princeton University Press, Princeton, N. J., 1951.

[29] M. Schiffer and G. Szegö, Virtual mass and polarization. Trans. Amer. Math. Soc., 67 (1949), 130-205. 
LMV, Université de Versailles St-Quentin, CNRS, Versailles, France.

E-mail address: Yves.capdeboscq@uvsq.fr

Department of Mathematical Sciences, Seoul National University, Seoul 151-747, Korea.

E-mail address: hbkang@snu.ac.kr 